\title{
Rotary-wing MAV Modeling \& Control for indoor scenarios
}

\author{
A. Barrientos, Senior Member, IEEE, J. Colorado, A. Martinez, Members, IEEE, and João Valente
}

\begin{abstract}
This paper is about modeling and control of Miniature Aerial Vehicles -MAVs for indoor scenarios, specially using, micro coaxial and quadrotor systems. Mathematical models for simulation and control are introduced and subsequently applied to the commercial aircraft: the DraganFlyer quadrotor and the Micro-Mosquito coaxial flying vehicle. The MAVs have been hardware-modified in order to perform experimental autonomous flight. A novel approach for control based on Hybrid Backstepping and the Frenet-Serret theory is used for attitude stabilization (Backstepping+FST), introducing a desired attitude angle acceleration function dependent on aircraft velocity. Results of autonomous hovering and tracking are presented based on the scheme we propose for control and attitude stabilization when MAV is maneuvering at moderate speeds.
\end{abstract}

\section{INTRODUCTION}

$\mathrm{R}$ ecent progress in sensor technology, data processing, and integrated actuators has made the development of Micro Aerial Vehicles -MAVs fully possible [1]-[3]. Depending on the flying principle and the propulsion mode, MAV can be classified into multiple categories: fixed, flapping, morphing and rotary wings are the most common mechanisms developed [4]-[6]. As usual, the real "Micro" mechanisms (at few centimeter-scale) have a span less than $15 \mathrm{~cm}$ with a total weight less than 50 grams, and equipped with MEMS sensors and piezoelectric actuators [7], [8].

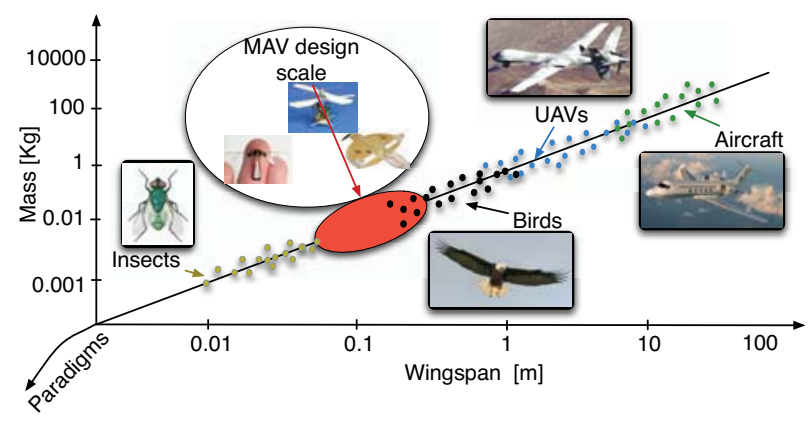

Fig. 1. Aerial Vehicles design-scale.

Depending of the size of the MAV (see Fig. 1), researchers focus on different phenomena and new paradigms and challenges related to mechanical design, electronic miniaturization [9], and new techniques for gaining more level of autonomy, which also depends on the MAV flying principle. In this sense we can observe that MAVs can be categorized in two categories: 1)-Micro or 2)Miniature flying vehicles. In the first category, scientist focus on researching how to approach from biologically inspired -at-scale- robot insects [4],[7], in which the deal is related to understand flying aerodynamics (at extremely low Reynolds numbers), and also developing/integrating electronics at that scale of design. However MAVs of the size of a small bird or even an insect are not still capable of autonomy or even 6-DoF flight, whereas the second category provides the ideal platform (due to more payload capacity and size) for testing methodologies to achieve full autonomous navigation [10],[11]. Despite nowadays the design of those mini aerial robots is widely extended, the full control is still a challenging goal.

In this sense, the aim of this paper is to introduce a novel control methodology named: Backstepping $+F S T$ to achieve reliable indoor autonomous navigation when MAV maneuvers at high speeds (up to $2 \mathrm{~m} / \mathrm{s}$ ). Simulation and experimental testing are conducted on Vertical Take-off and Landing -VTOL systems (like rotary-wing vehicles) because of their unquestionable advantage compared to other concepts in terms of the ability for vertical and stationary flight, but really challenging in relation to control.

\section{PROBLEM APPROACH}

In terms of control, the attitude control of a MAV is crucial [12]. It provides the required stabilization to perform aggressive maneuvering and reliable navigation maintaining 3D orientation. Classical control (e.g. PID) applied to attitude stabilization has being used for awhile [13], however, due to its design, rotary-wing MAVs are unable to move in an uncoupled way, and as a result of this underactuation, standard control techniques do not work well on these crafts. On the other hand, most works [14]-[17] whether use non-linear control techniques to improve on the autonomous flight, but despite the substantial interest of studying dynamics nonlinearities, and design methodologies, little attention has been paid to improve on the attitude tracking based on the velocity and acceleration of the aircraft during flight.

From a modeling perspective, both rotary-wing: the DraganFlyer and the coaxial Micro-Mosquito (see Fig. 2) have highly coupled dynamics: a change in the speed of one rotor results in motion in at least 2-DoF. For example (in the case of the DraganFlyer), reducing the speed of the right rotor will cause the craft to roll to the right due to the imbalance between left and right lift forces. In addition, its small size, highly coupled dynamics, low air drag on the fuselage and high air drag on the rotors, pose significant challenges in the control of this MAV. 
On the other hand, coaxial structure provides enhancements related to stability and controllability due to it uses two contra-rotating rotors to compensate the torque that they apply to the fuselage when rotating. In addition a tail rotor provides to the MAV with pitch control. Nonetheless, its payload capacity (25 grams) constrains the MAV to address powerful sensing capabilities, which consequently require reinforcing the control approach.

From this analysis it becomes clear that reliable attitude stabilization is required to achieve the desired level of performance during flying. To improve on the attitude control under these characteristics, this work focuses on applying a hybrid Backstepping nonlinear control technique and the Frenet-Serret Theory-FST [18] (Backstepping+FST) that includes estimation of the desired angular acceleration as a function of the aircraft velocity, improving MAV maneuvering in presence of abrupt angular rate changes.

\section{MAV SYSTEM MODELING}

This section deals with the description of the fundamental concepts related to the rigid body dynamics modeling, presenting the Equations of Motion -EoM using the spatial operator algebra [19] applied to: The DraganFlyer [20], and the Micro-Mosquito [21].

\section{A. System Description}

The DraganFlyer is a radio-controlled four-rotor aerial vehicle with four channels of input to control the motion of the MAV. Varying the speed of the four rotors the motion of DraganFlyer can be controlled (propellers $(1,3)$ and $(2,4)$ turn in opposite directions, see Fig. 2a).

On the other hand, the Micro-Mosquito in Fig. 2b is a coaxial mechanism that flies up, down, forward, reverse, turn left and right with 3 channel digital proportional control. In the coaxial configuration, one propeller is located above the other with a common shaft.

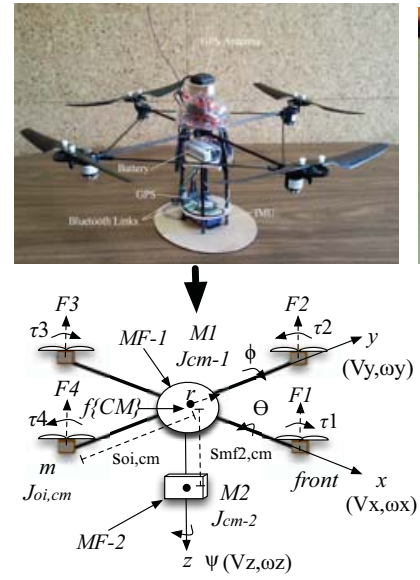

a)

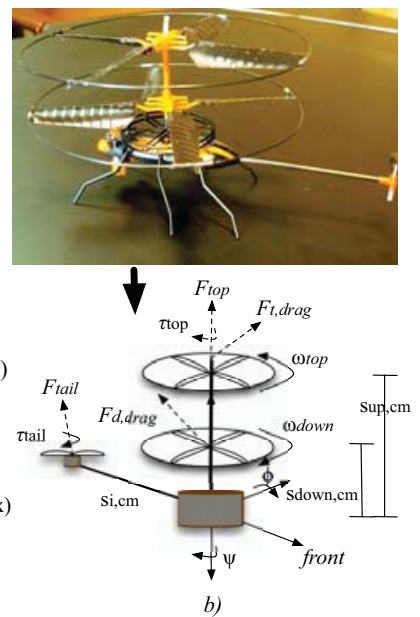

b)
Fig. 2. MAV System Description for Modeling.

The rotors turn in opposite directions, which removes the need for a tail rotor (for torque compensation purposes), and makes the helicopter a lot more compact. Typical coaxial MAVs use the residual torque due to angular speed difference between the two rotors to rotate the helicopter vertically, left or right. Increasing or decreasing the angular speed of the rotors simultaneously permits climbing and descending.

\section{B. Dynamics Equations of Motion}

Assuming from Fig. $3 \mathrm{~b}$ that $O_{i}$ and $\mathrm{CM}$ are two points located on the rigid body, and $s_{o i, c m} \in \mathfrak{R}^{3}$ is the vector that joints the extreme border $O_{i}$ with the rigid body's center of mass, the translational and angular velocities $(v, \omega)$ and forces $(f, \tau)$ respectively at any point on a body in $\mathfrak{R}^{3}$ are related as shown in (1). In terms of spatial algebra, the physical quantities are represented as $6 \times 1$ column vectors, in which Euler parameterization is used for kinematics transformation. Equation 1 shows the DraganFlyer model:
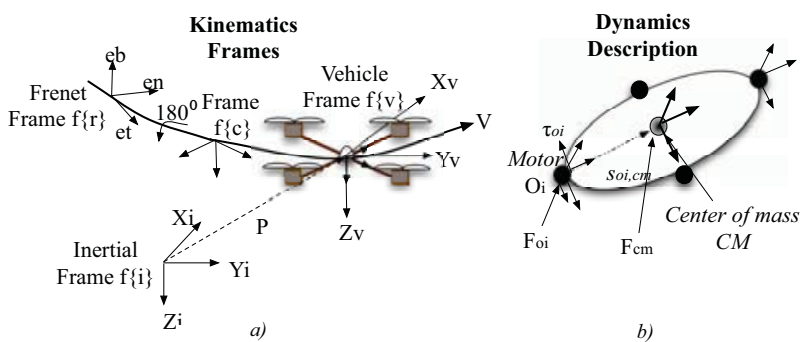

Fig. 3. MAV System Description for Modeling.

$$
\begin{aligned}
\dot{V}_{c m} & =\left[\begin{array}{c}
\dot{\omega}_{c m} \\
\dot{v}_{c m}
\end{array}\right]=I_{c m, T}^{-1}\left[F_{c m, T}-\dot{I}_{c m, T} V_{c m}\right] \\
& =\left[\begin{array}{cc}
J_{c m, T}-\tilde{s}_{o i, c m} m_{T} \tilde{s}_{o i, c m} & m_{T} \tilde{s}_{o i, c m} \\
-m_{T} \tilde{s}_{o i, c m} & m_{T} U
\end{array}\right]^{-1}\left[\begin{array}{c}
\tau_{c m, T}-\tilde{\omega}_{c m} J_{c m, T} \tilde{\omega}_{c m} \\
f_{c m, T}
\end{array}\right]
\end{aligned}
$$

The term $J_{c m, T} \in \mathfrak{R}^{3 \times 3}$ in (2) indicates the total inertia of the vehicle due to rotors and electronics with total mass $m_{T}$ :

$$
J_{c m, T}=\frac{2}{5} M_{1} r^{2}+M_{2}\left(\frac{a^{2}+b^{2}}{12}+s_{m f 2, c m}^{2}\right)+m\left(r_{m}^{2}+s_{o i, c m}^{2}\right)
$$

For the DraganFlyer, spherical-shape has been adopted from main electronics with mass $M_{1}$ and radius $r$, whereas rectangular with mass $M_{2}$ and cylindrical $\left(m, r_{m}\right)$ shapes for second electronics (battery + IMU) and the 4-rotors respectively. The terms $(a, b)$ refer to the lengths of the rectangular shape assumed for second electronics (see Fig.2a).

$$
\dot{V}_{c m}=\left[\begin{array}{cc}
J_{c m, T}-\tilde{s}_{o i, c m} m \tilde{s}_{o i, c m} & m \tilde{s}_{o i, c m} \\
-m \tilde{s}_{o i, c m} & m U
\end{array}\right]^{-1}\left(\left[\begin{array}{c}
\tau_{c m, T} \\
f_{c m, T}
\end{array}\right]-\left[\begin{array}{c}
\xi_{T} \\
0
\end{array}\right]\right)
$$


On the other hand, the term $\tilde{s}_{o i, c m} \in \mathfrak{R}^{3 \times 3}$ is the skew symmetric matrix corresponding to the vector cross product operator of $s_{o i, c m}$ (see Fig. 3 for operators description), and finally $U \in \mathfrak{R}^{3 \times 3}$ refers to the identity operator. In the same way, (4) shows the Micro-mosquito dynamics model, being the velocity-dependent force term $\xi_{T}$ :

$$
\begin{aligned}
\xi_{T}= & \tilde{s}_{t o p, c m}\left(\tilde{\omega}_{\text {top }} J_{c m, T} \tilde{\omega}_{\text {top }}\right)+\tilde{s}_{\text {down }, c m}\left(\tilde{\omega}_{\text {down }} J_{c m, T} \tilde{\omega}_{\text {down }}\right)+ \\
& \tilde{s}_{o i, c m}\left(\tilde{\omega}_{\text {tail }} J_{c m, T} \tilde{\omega}_{\text {tail }}\right)
\end{aligned}
$$

Where the total inertia term $J_{c m, T}$ corresponds:

$$
J_{c m, T}=\frac{R_{p}^{2}}{4}+\frac{e_{p}^{2}}{12}\left(s_{u p, c m}^{2}+s_{d o w n, c m}^{2}\right)+m \frac{a^{2}+b^{2}}{12}
$$

The parameters $R_{p}$ and $e_{p}$ refer to the radius and thickness of the disk-shape formed by propeller rotation. Terms $(a, b)$ to the lengths of a rectangle-box shape assumed for electronics onboard. In addition the Table-I shows the electronics component description of both MAV.

TABLE I MAV ELECTRONICS COMPONENT DESCRIPTION

\begin{tabular}{ll}
\multicolumn{1}{c}{ DraganFlyer } & \multicolumn{1}{c}{ Micro-Mosquito } \\
\hline \hline Width: $0.63 \mathrm{~m}$ & Length: $18.5 \mathrm{~cm}$ \\
Rotor span: $0.35 \mathrm{~m}$ & Control: $3 \mathrm{ch}$. digital FM \\
Main battery: 92 grams & $\begin{array}{l}\text { Motors: } 7 \mathrm{~mm} \text { coreless }+4 \\
\text { mm for tail. }\end{array}$ \\
& Battery: $160 \mathrm{mAh}$ li-po \\
IMU: 40 grams & Flight time: about $10 \mathrm{~min}$ \\
Bluetooth card-1: 10 grams & Rotor span: $16 \mathrm{~cm}$ \\
GPS: 17 grams & Height: $8 \mathrm{~cm}$ \\
Antenna: 45 grams & \\
Voltage Converter: 25 grams & \\
Bluetooth card-2: 7 grams & \\
Mainframe: 25 gram & \\
Total weight: 686 grams & Total weight: 20 grams
\end{tabular}

\section{AutONOMOUS FLIGHT}

For achieving full autonomous flight, two main modules compose the architecture for modeling and control: The System Modeling (previously introduced in section-III), and The System control (see Fig. 4). In terms of control, mapping the commands from control space to force space requires a model of the forces and their interactions. In the case of the DraganFlyer, each motor produces a force $(F)$ and torque $(\tau)$. For the rotational force-components, the rolling torque is produced by the forces of the right and left motors: $\tau_{2}$ and $\tau_{4}$, similarly the pitching torque is produced by the forces of the front and back actuators: $\tau_{3}$ and $\tau_{1}$. For the MicroMosquito pitching torque is proportional to tail lift force and yawing torque is proportional to the difference in rotation of main propellers.

During the development of this project we explored several control methodologies from theoretical development to final experiments. As a first attempt, we tested on DraganFlyer a PID controller based on a simplified

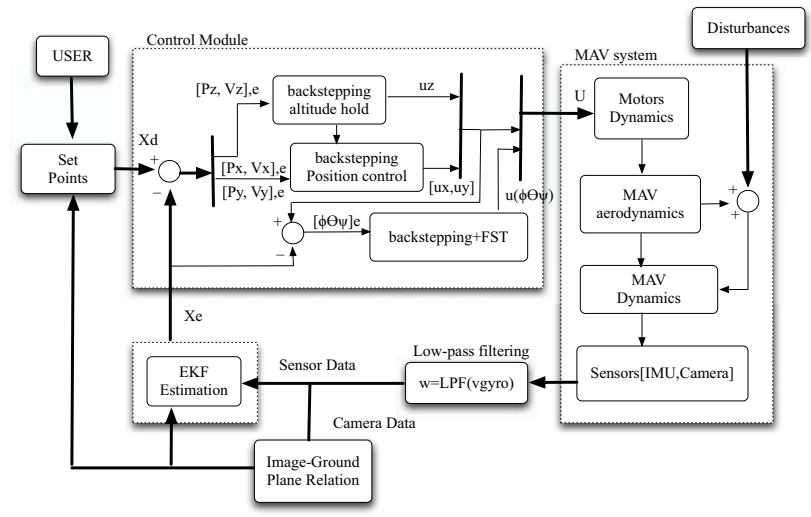

Fig. 4. Architecture for MAV autonomous flight.

model, however strong disturbances were poorly rejected. In the second attempt we reinforced the control using backstepping technique. This time, simulation and experimental results confirmed improvements in relation to disturbance rejection.

The backstepping technique has been used for some time for controlling quadrotors [10]. Improvements have been introduced thanks to combine integral action within the control law (integral-backstepping), which consequently asymptotic stability is guaranteed as well as steady state errors cancelation due to integral action. Nonetheless, poor analysis has been conducted on specifically improving attitude control, while the aircraft is maneuvering at moderate speeds and performing aggressive changes in orientation. To improve on this, we have adopted the FrenetSerret formulation used in vector calculus to describe the kinematic properties of the aircraft that moves along a continuous and differentiable curve in the Euclidian space. Consequently, improvements on attitude stabilization using integral-backstepping+FST as a function of a desired aircraft acceleration command are achieved. Next section explains this issue in detail.

\section{A. System Control}

The complete system control is composed by a cascadeconnection of altitude, position and attitude controllers (see Fig. 4). Attitude control is the heart of the control system that maintains the MAV stable and oriented towards the desired direction. This section shows roll-control derivation based on hybrid backstepping and the Frenet-Serret equations previously introduced. Note that for both pitch and yaw-control the same methodology is used. Based on the dynamics model from (1), the first step is considering the roll tracking error of the DraganFlyer $e_{1}$ and its derivative with respect to time.

$$
\begin{gathered}
e_{1}=\phi^{d}-\phi \\
\dot{e}_{1}=\dot{\phi}^{d}-\omega_{x}
\end{gathered}
$$


A Lyapunov function (positive definite) is used for stabilizing the tracking error $e_{1}$, based on a virtual control law for setting the desired behavior of the angular speed $\omega_{x}$ :

$$
\begin{aligned}
& V\left(e_{1}\right)=\frac{e_{1}^{2}}{2} \\
& \dot{V}\left(e_{1}\right)=e_{1}\left(\dot{\phi}^{d}-\omega_{x}\right)
\end{aligned}
$$

The virtual control law for stabilizing the angular tracking error $e_{2}$ is then defined as:

$$
\begin{aligned}
& e_{2}=\omega_{x}^{d}-\omega_{x} \\
& \omega_{x}^{d}=c_{1} e_{1}+\dot{\phi}^{d}+\lambda_{1} \int e_{1}
\end{aligned}
$$

Replacing the $\omega_{x}^{d}$ term in (8), and deriving $e_{2}$ with respect to time:

$$
\begin{aligned}
& e_{2}=c_{1} e_{1}+\dot{\phi}^{d}+\lambda_{1} \int e_{1}-\omega_{x} \\
& \dot{e}_{2}=c_{1}\left(\dot{\phi}^{d}-\omega_{x}\right)+\ddot{\phi}^{d}+\lambda_{1} e_{1}-\ddot{\phi}
\end{aligned}
$$

Now replacing $\omega_{x}$ from (8) into $\dot{e}_{1}$ in (6), and then replacing $\dot{e}_{1}$ into $\dot{e}_{2}$ in (9):

$$
\begin{aligned}
& \dot{e}_{1}=-c_{1} e_{1}-\lambda_{1} \int e_{1}+e_{2} \\
& \dot{e}_{2}=c_{1}\left(-c_{1} e_{1}-\lambda_{1} \int e_{1}+e_{2}\right)+\ddot{\phi}^{d}+\lambda_{1} e_{1}-\ddot{\phi}
\end{aligned}
$$

Extracting from (1) the dynamics terms corresponding to angular acceleration $\ddot{\phi}$ of the DraganFlyer, and replacing them into (10):

$$
\begin{aligned}
\dot{e}_{2}= & c_{1}\left(-c_{1} e_{1}-\lambda_{1} \int e_{1}+e_{2}\right)+\ddot{\phi}^{d}+ \\
& \lambda_{1} e_{1}-J_{x-c m, T}^{-1}\left[\dot{\theta} \dot{\psi}\left(J_{y-c m, T}-J_{z-c m, T}\right)+\tau_{\phi}\right]
\end{aligned}
$$

Solving (11) for $\tau_{\phi}$ which is the control law for achieving roll stabilization, and defining the desirable dynamics for the angular speed tracking error as $\dot{e}_{2}=-e_{1}-c_{2} e_{2}$ :

$$
\begin{aligned}
& \tau_{\phi}=J_{x-c m, T} \Omega+\xi \\
& \Omega=e_{1}\left(c_{1}^{2}-1-\lambda_{1}\right)-e_{2}\left(c_{1}+c_{2}\right)+c_{1} \lambda_{1} \int e_{1}-\ddot{\phi}^{d} \\
& \xi=\dot{\theta} \dot{\psi}\left(J_{y-c m, T}-J_{z-c m, T}\right)
\end{aligned}
$$

Finally, the term $\ddot{\phi}^{d}$ in (12) is replaced by the desired angular acceleration command to be obtained with the Ferret theory. To understand how to obtain this term, refer to Fig. 3a in which the different frames used to operate the EoM were introduced. The Vehicle-frame $\mathrm{f}\{\mathrm{v}\}$ and the
Inertial-frame $\mathrm{F}\{\mathrm{i}\}$ that are related with each other using Euler parameterization, and two additional frames called the Frenet-frame $f\{r\}$ and the rotated Frenet-frame $f\{c\}$ which are composed by three unit vector so-called the tanget $\left(e_{t}\right)$, normal $\left(e_{n}\right)$, and binormal $\left(e_{b}\right)$, that move along the curve or desired trajectory. Imagine that an observer moves along the curve in time, using the attached frame at each point as its coordinate system. The Frenet-Serret formulas mean that this coordinate system is constantly rotating as an observer moves along the curve; hence, this coordinate system is always non-inertial. So, the position and the magnitude of the velocity vector at any point of the trajectory are given by:

$$
V=\|\dot{P}\|=\sqrt{\dot{P}_{x}^{2}+\dot{P}_{y}^{2}+\dot{P}_{z}^{2}}
$$

To every point of the curve we can associate an orthonormal triad of vectors namely the tangent, the normal and the bio-normal (see Fig. 3a). Properly arranging these vectors, we obtain a description of the curve orientation. The corresponding reference frame is the Frenet-Serret $\mathrm{f}\{\mathrm{r}\}$ one.

$$
e_{t}=\frac{\dot{P}}{V}, \quad e_{b}=\frac{(\dot{P} \times \ddot{P})}{\|\dot{P} \times \ddot{P}\|}, \quad e_{n}=e_{b} \times e_{t}
$$

As far as the reference orientation $\left[\phi_{R}, \theta_{R}, \psi_{R}\right]^{T}$ of the body-fixed frame $\mathrm{f}\{\mathrm{v}\}$ with respect to the inertial $\mathrm{f}\{\mathrm{i}\}$ frame is concerned, due to the dynamics, $f\{v\}$ does not coincide with the $f\{c\}$ frame. To eventually coincide with the reference desired frame $f\{R\}$ that provides the orientation consistent with the aircraft dynamics, the rotation of the $f\{v\}$ frame from $\mathrm{f}\{\mathrm{c}\}$ to $\mathrm{f}\{\mathrm{R}\}$ can be expressed using customary aeronautical notation by considering the sideslip angle $\beta$, and the angle of attack $\alpha$ :

$$
\begin{aligned}
& \beta=\sin ^{-1}\left(\frac{\dot{P}_{y}}{V}\right), \alpha=\tan ^{-1}\left(\frac{\dot{P}_{z}}{\dot{P}_{x}}\right) \\
& R_{\{c\}}^{\{R\}}=R_{y}^{T}(\alpha) R_{z}^{T}(-\beta) \\
& R_{\{i\}}^{\{R\}}=R_{\{c\}}^{\{R\}} R_{\{c\}}^{\{i\}}
\end{aligned}
$$

Using the second derivative of $R_{\{i\}}^{\{R\}}$ with respect to time, the angular acceleration references can be extracted from matrix components as:

$$
\begin{aligned}
& \ddot{\phi}^{d}=\frac{d^{2}}{d t^{2}}\left(\operatorname{atan} 2\left(R_{\{i\}, 23}^{\{R\}}, R_{\{i\}, 33}^{\{R\}}\right)\right) \\
& \ddot{\theta}^{d}=\frac{d^{2}}{d t^{2}}\left(\operatorname{atan} 2\left(-R_{\{i\}, 13}^{\{R\}}, \sqrt{\left(R_{\{i\}, 23}^{\{R\}}\right)^{2}+\left(R_{\{i\}, 33}^{\{R\}}\right)^{2}}\right)\right) \\
& \ddot{\psi}^{d}=\frac{d^{2}}{d t^{2}}\left(\operatorname{atan} 2\left(R_{\{i\}, 12}^{\{R\}}, R_{\{i\}, 11}^{\{R\}}\right)\right)
\end{aligned}
$$

Replacing $\ddot{\phi}^{d}$ from (16) into (12), the Backstepping+FST 
control is presented. Next section presents experimental and simulation results conducted on both MAV platforms.

\section{RESUlts}

In order to validate that our assumption of improving attitude stabilization within the Backstepping+FST is indeed correct, Fig. 5 and 6 show the experimental results for the first test performed using the DraganFlyer MAV. Control parameters used in this experiment are: for roll: $\left[c_{1}, c_{2}, \lambda_{1}\right]=[5.5,0.5,0.01]$, pitch: $\left[c_{3}, c_{4}, \lambda_{2}\right]=[10,2,0.01]$, yaw: $\left[c_{5}, c_{6}, \lambda_{3}\right]=[2,1.5,0.005]$.

Note in Fig. 6 the PID controller proved to be well adapted to the MAV when flying near to hover. For this kind of test (hovering control), there are not huge differences of using the backstepping+FST control against single backstepping or either PID controllers. Note that just a slight difference in relation to amplitude and time oscillation is improved with the backstepping+FST. The reason is that this controller has been designed to improve on the attitude stabilization when aircraft is maneuvering at moderate speed. To take advantage of the backstepping+FST, the trajectory of the aircraft must be smooth enough (e.g. threetimes differentiable with respect to time) in order to achieve the desired values of attitude angles based on the references.

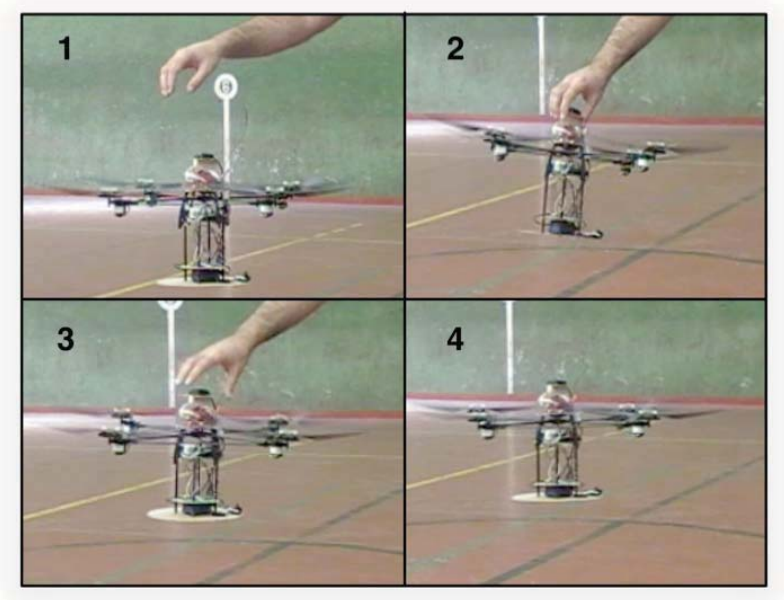

Fig. 5. Strong external disturbances addressed during experiment in Fig. 6.
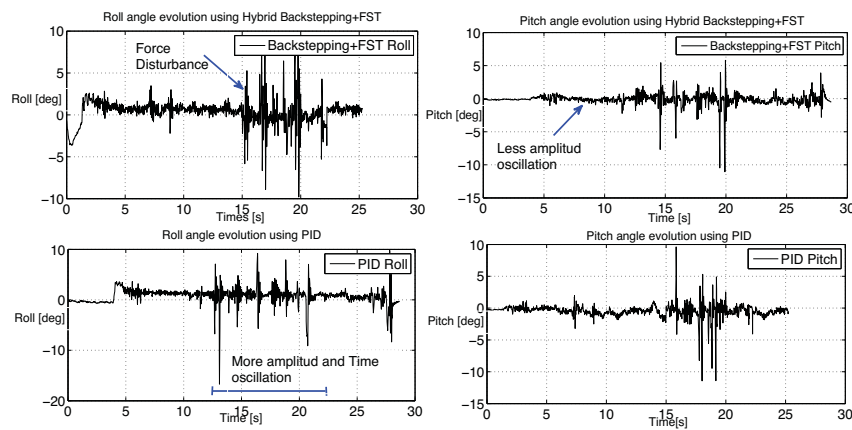

Fig. 6. Attitude control: Comparison between backstepping+FST control against PID controller while maintaining aircraft attitude angles to zero.

To achieve full indoor autonomous navigation (altitude and position), a camera must be placed on the MAV. For instance, the DraganFlyer platform has just been hardwaremodified by addressing IMU and GPS for outdoor navigation. Our final goal on this project is to address the camera onboard for both DraganFlyer and Micro-Mosquito in order to perform tracking tasks based on vision.

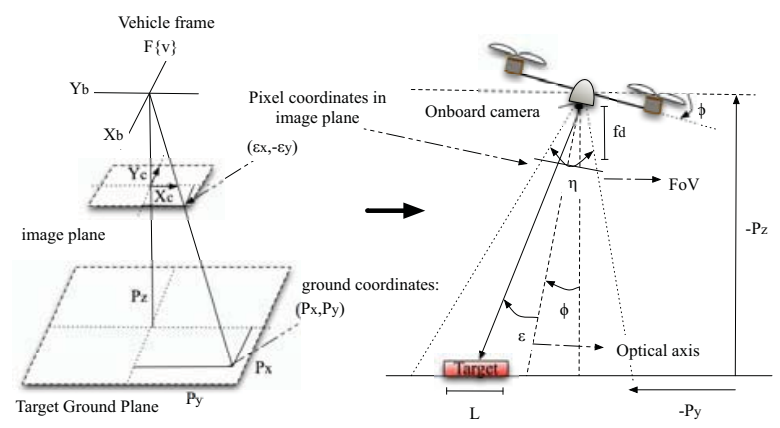

Fig. 7. Camera model for indoor navigation

The control objective is to maintain the vehicle in a constant altitude $\left(P_{z}\right)$ while tracing the horizontal trajectory defined by the target on ground (see Fig. 7). For altitude and position control (same backstepping approach used in (11) is adopted), and camera information based on relating the real size of the target $(L)$, the size of the target projected in the image plane $\varepsilon$ (given in pixels), the focal distance $f d$ and the altitude to hold $P_{z}$, are used for defining the control laws.

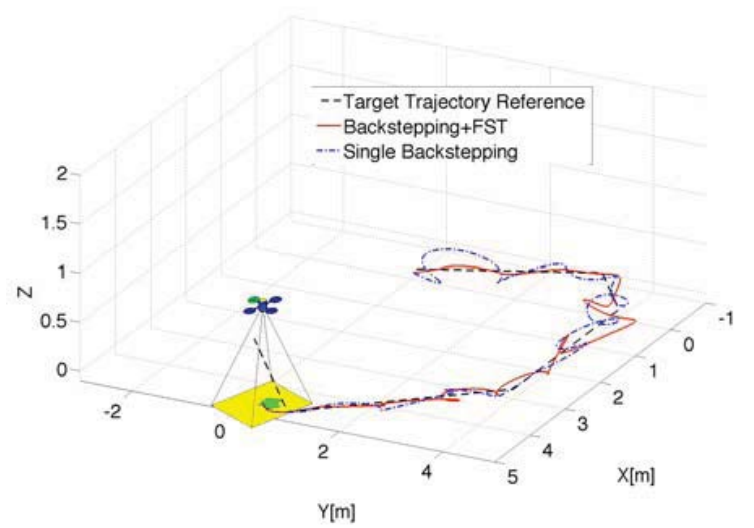

Fig. 8. DraganFlyer full navigation simulation experiment: indoor navigation based on tracking a target on ground up to speeds of $2 \mathrm{~m} / \mathrm{s}$ (including wind disturbances of $0.5 \mathrm{~m} / \mathrm{s}$ in both $x-y$ axes).

From Fig. 8 note that target's trajectory profile performs four-aggressive orientation changes (yaw-angle) during tracking at linear speed of $2 \mathrm{~m} / \mathrm{s}$. The DraganFlyer perfectly tracked the target maintaining the orientation given by the target at $1[\mathrm{~m}]$-altitude from ground. Because of the angular acceleration estimation introduced in the Backstepping +FST, attitude stabilization based on the aircraft velocity profile improves on the tracking error (in X-Y position) of the quadrotor while tracking the target. In addition, external disturbances are poorly rejected using the single 
backstepping approach against the FST inclusion.

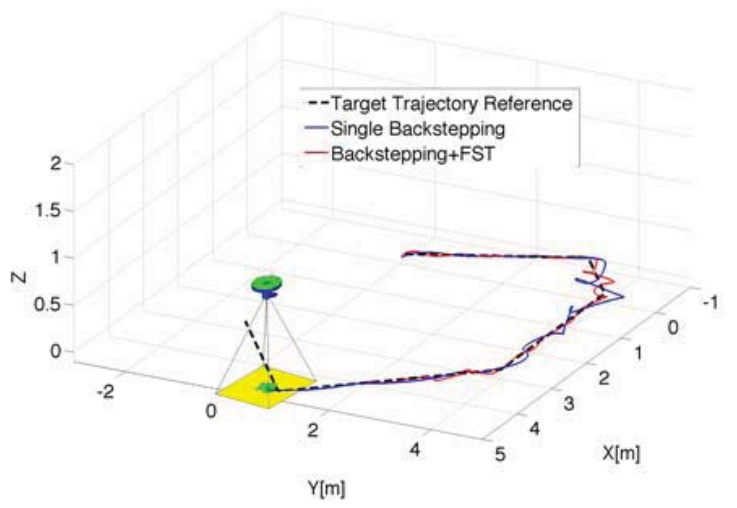

Fig. 9. Micro-Mosquito full navigation simulation experiment: indoor navigation based on tracking a target on ground up to speeds of $2 \mathrm{~m} / \mathrm{s}$.

Backstepping+FST is being applied to the MicroMosquito MAV. As shown in Fig. 9, improvements on attitude stabilization also improves on position tracking compared to single Baskstepping approach. In this case, note that the coaxial mechanism is more stable during flight compared to the quadrotor system.

\section{CONCLUSIONS}

A hybrid Backstepping+FST methodology has been proposed for attitude control for MAV autonomous navigation. Experimental results performed in Fig. 5 and 6 showed how the control maintains attitude references in presence of external disturbances.

For full indoor navigation, Future work includes addressing vision capability to the DraganFlyer and the Micro-Mosquito, and finally testing position and altitude control beyond simulation. Nonetheless, results obtained in Fig. 8 and 9 are motivating. At high speed maneuvering $(2 \mathrm{~m} / \mathrm{s})$, the backstepping+FST's performance (in relation to error tracking) is about $3.5 x$ times better than using the single backstepping technique (in the case of the DraganFlyer). For the Micro-Mosquito, the tracking is even more reliable thanks to the advantage related to the stabilization presented on coaxial mechanisms.

For both cases, the improvement in tracking was basically achieved by introducing a desired angular acceleration command (as a function of the maneuvering velocity) that quickly responds to abrupt angular rate change, making the attitude stabilization more reliable.

\section{ACKNOWLEDGMENT}

This work was supported by the Robotics and Cybernetics Group at Technique University of Madrid, Spain and funded under the project FRACTAL: Fleet of cooperative terrestrial and aerial robots, sponsored by the Spain Ministry of Education and Science (DPI 2006-03444) and ROBOCITY 2030 (S-0505/DPI/ 000235), http://www.robocity2030.org/

\section{REFERENCES}

[1] Zufferey, J.-C., Klaptocz, A., Beyeler, A., Nicoud, J.-D., and Dario. A 10-gram Microflyer for Vision-based Indoor Navigation. Proceedings of the IEEE/RSJ International Conference on Intelligent Robots and Systems, Beijing, China, 2006, p. 3267-3272.

[2] Wood, R. Design, fabrication, and analysis of a $3 \mathrm{DOF}, 3 \mathrm{~cm}$ flappingwing MAV. Proceedings of the IEEE/RSJ International Conference on Intelligent Robots and Systems, San Diego, CA, USA, 2007.

[3] Bouabdallah, S., Becker, M., and Siegwart, R. Autonomous miniature flying robots: coming soon!. IEEE Robotics \& Automation Magazine, 2007, vol. 14 (3), 2007, p88 - 98.

[4] Madangopal R., Khan Z., and Agrawal S. Biologically inspired design of small flapping wing air vehicles using four-bar mechanisms and quasi-steady aerodynamics. Journal of Mech. Design, vol. 127, pp. 809-816, July 2005.

[5] Bouabdallah, S., Siegwart, R, and Caprari, G., Design and Control of an Indoor Coaxial Helicopter. Proceedings of the IEEE/RSJ International Conference on Intelligent Robots and Systems, Beijing, China, 2006 pp. $2930-2935$.

[6] Bachmann, R., Boria, F., Ifju, P., Quinn, R., Kline, J., and Vaidyanathan, R. Utility of a Sensor Platform Capable of Aerial and Terrestrial Locomotion. Proceedings of the IEEE/ASME International Conference on Advanced Intelligent Mechatronics, Monterey, California, USA, 2005.

[7] Zufferey J-C., and Floreano D., Fly-inspired visual steering of an ultralight indoor aircraft, IEEE Transactions on Robotics, vol. 22, no. 1, pp. 137-146, 2006.

[8] Steltz, E., Seeman, M., Avadhanula, S., and Fearing, R. (2006, Jul 2). Power Electronics Design Choice for Piezoelectric Microrobots. Proceedings of the IEEE/RSJ International Conference on Intelligent Robots and Systems, Beijing, China, 2006.

[9] Karpelson, M., Gu-Yeon Wei, and Wood, R. A review of actuation and power electronics options for flapping-wing robotic insects. The IEEE International Conference on Robotics and Automation, Pasadena, CA, USA, 2008, pp. 779 - 786.

[10] Bouadi, H., Bouchoucha, M., \& Tadjine, M. Sliding Mode Control based on Backstepping Approach for an UAV Type-Quadrotor. Proceedings of world academy of science, engineering and technology, Vol. 20, 2007.

[11] Waslander, S., and Hoffmann, G. Multi-Agent Quadrotor Testbed Control Design: Integral Sliding Mode vs. Reinforcement Learning. The IEEE/RSJ International Conference on Intelligent Robots and Systems, Alberta, Canada, 2005.

[12] Olfati-Saber, R. Nonlinear Control of Underactuated Mechanical Systems with Application to Robotics and Aerospace Vehicles. PhD thesis, Massachusetts Institute of Technology, 2001.

[13] Bouabdallah S., Noth A. PID vs LQ control techniques applied to an indoor micro quadrotor. Proceedings of the IEEE/RSJ International Conference on Intelligent Robots and Systems. Sendai, Japan, 2004.

[14] Zufferey, J.-C., Beyeler, A., and Floreano, D. Optic flow to control small UAVs. Proceedings of the IEEE/RSJ International Conference on Intelligent Robots and Systems, Nice, France, 2008.

[15] Bouabdallah, S., and Siegwart, R. Full Control of a Quadrotor. Proceedings of the IEEE/RSJ International Conference on Intelligent Robots and Systems, San Diego, CA, USA, 2007.

[16] Mokhtari, A. and Benallegue, A,. Dynamic Feedback Controller of Euler Angles and Wind parameters estimation for a Quadrotor Unmanned Aerial Vehicle. Proceedings of the IEEE International Conference on Robotics and Automation, New Orleans, LA, April 2004, pp. 2359-2366.

[17] Altug E., Ostrowski, J. P. and Mahony R. Control of a Quadrotor Helicopter Using Visual Feedback, Proceedings of the IEEE International Conference on Robotics and Automation, 2002.

[18] Hanson, A. Quaternion Frenet Frames: Making Optimal Tubes and Ribbons from Curves. Indiana University Technical Report, 1-9, 2007

[19] Rodriguez G., Jain A., and Kreutz-Delgado K. Spatial operator algebra for manipulator modeling and control, Int. Journal of Robotics Research. 10(4) (1991), 371-381.

[20] The DraganFlyer. http://www.draganfly.com

[21] The Micro-Mosquito. http://rctoys.com/

[22] Wayne J. Helicopter Theory, Princeton University Press, Princeton, New Jersey, 1980. 